\title{
Optical-field-ionization EUV lasing in a xenon cluster jet
}

\author{
Hsu-hsin Chu \\ Department of Physics, National Taiwan University, Taipei 106, Taiwan \\ Hai-En Tsai \\ Graduate Institute of Electro-Optical Engineering, National Taiwan University, Taipei 106, Taiwan \\ Jiunn-Yuan Lin \\ Department of Physics, National Chung Cheng University, Chia-Yi, Taiwan \\ Chau-Hwang Lee \\ Research Center for Applied Sciences, Academia Sinica, Taipei 115, Taiwan \\ Lan-Sheng Yang, Jyhpyng Wang and Szu-yuan Chen \\ Institute of Atomic and Molecular Sciences, Academia Sinica, Taipei 106, Taiwan \\ Tel: 886-2-2366-8276, Fax: 886-2-2362-7064, sychen@ltl.iams.sinica.edu.tw
}

\begin{abstract}
An optical-field-ionization EUV laser with prepulse-controlled nanoplasma expansion in a cluster gas jet was demonstrated. Pd-like xenon lasing at 41.8-nm with $100 \mathrm{~nJ} / \mathrm{pulse}$ and 5mrad divergence was achieved, indicating near-saturation amplification. (C)2005. Optical Society of America OCIS codes: $(000.0000)$ General
\end{abstract}

Collision-excitation EUV lasing by optical-field-ionization (OFI) heating was first demonstrated in 1995 at $41.8 \mathrm{~nm}$ with a xenon gas cell [1]. Saturated amplification was later achieved [2] and lasing at shorter wavelength was obtained with krypton [3]. Two important breakthroughs in lasing efficiency were reported recently: one is amplification in a preformed plasma waveguide driven by discharge in a capillary tube filled with xenon and buffer gas [4], and the other is seeding of the amplifier with pulses from high-harmonic generation [5]. Here we report the first demonstration of an OFI EUV laser using a cluster jet. Comparing to gas cells or capillary tubes, gas-jet targets have some advantages: (1) more flexible in controlling the density, (2) no damage or contamination problems, and (3) the average gas flow is small, hence is more cost-effective for continuous operation using expensive rare gases. In order to produce a uniform gas jet with sharp boundaries, supersonic gas flow is used. For heavy atoms it is known that clusters are formed in supersonic jets [6], therefore, how to achieve soft x-ray amplification in cluster jets needs to be explored. To make a uniform plasma out of gas clusters, we use a prepulse to ionize and heat up the clusters. By controlling the expansion of the clusters with the prepulse intensity and the delay between the prepulse and the pump pulse, it is shown that the uniformity of the nanoplasma gas is essential for efficient lasing, and the optimal atom density for maximum lasing reported previously $[2,3]$ is a result of balance between large gain coefficient and short gain length due to ionization-induced refraction.

A 10-TW, 55-fs, 810-nm, 10-Hz Ti:sapphire laser system [7] was used for this experiment. A prepulse and a pump pulse both with 55-fs duration propagates collinearly and are focused with a concave mirror of $1-\mathrm{m}$ focal length onto a xenon cluster jet. The jet has a flat-top region of $4.5 \mathrm{~mm}$ in length and the average diameter of the clusters is about $15-50 \mathrm{~nm}$ for a backing pressure of $7 \times 10^{5}-4 \times 10^{6} \mathrm{~Pa}$. The focal spot size of the $350-\mathrm{mJ}$ pump pulse is $25 \mu \mathrm{m}$, resulting in a peak intensity of $7 \times 10^{17} \mathrm{~W} / \mathrm{cm}^{2}$. The beam size of the prepulse in the jet is tuned to $200 \mu \mathrm{m}$ by a telescope. A spectrometer composed of an aperiodic grazing-incidence grating and a 16-bit imaging CCD camera is used to measure the EUV emission spectrum and divergence angle in the direction of laser propagation. The spectral range and resolution are 30-60 $\mathrm{nm}$ and $0.05 \mathrm{~nm}$ respectively. Aluminum filters are used to block transmitted laser pulses and attenuate EUV emission. By calibrating the grating reflectivity, filter transmittance, and CCD response, the absolute emission yield can be retrieved. Mach-Zehnder interferometry with a probe pulse passing transversely through the cluster jet is used to measure the evolution of the density distribution of plasma electrons. An imaging system is used to measure Rayleigh scattering from nanoplasmas, which time-resolves the expansion of the nanoplasmas.

Figure 1 shows the EUV emission spectra for an average atom density of $6 \times 10^{17} \mathrm{~cm}^{-3}$ and a pump-pulse delay of $3.8 \mathrm{~ns}$. The energies of the prepulse and pump pulse are $17 \mathrm{~mJ}$ and $350 \mathrm{~mJ}$, respectively. Strong lasing signal is observed at $41.8 \mathrm{~nm}$ when both pulses are circularly polarized. When the polarization is changed to linear, the lasing signal vanishes. Previous studies on above-threshold ionization (ATI) $[8,9]$ show that the electron energy 


\section{JThG5}

distribution after ionization by circularly-polarized laser pulses has a much higher temperature than that by linearlypolarized pulses. The polarization dependence in our experimental results indicates that the laser is pumped by optical-field ionization and ATI heating. The output energy reaches 100-nJ/pulse, which is larger than the saturated output energy reported previously with similar pump energy, atom density, and gain medium length [2]. The EUV beam divergence is 5-mrad, smaller than the aspect ratio of the plasma column. These data indicates that the amplification is close to saturation. When the prepulse is turned off, no lasing signal is observed for either polarization. In this case the continuum and the characteristic line emissions of various ionization stages are enhanced greatly, showing that the interaction of the pump pulse is dominated by collisional ionization and heating in nanoplasmas.

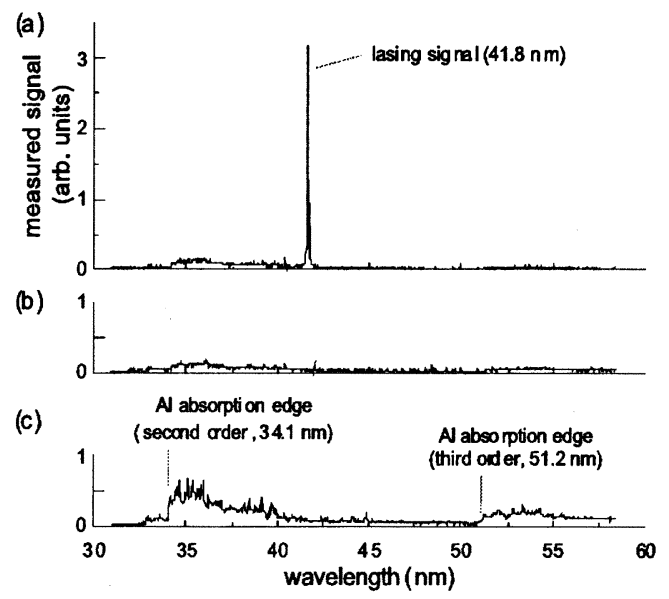

Fig. 1. Measured spectra for (a) a $17-\mathrm{mJ}$ prepulse and a $350-\mathrm{mJ}$ pump pulse of circular polarization, (b) a $17-\mathrm{mJ}$ prepulse and a $350-\mathrm{mJ}$ pump pulse of linear polarization, and (c) only a $350-\mathrm{mJ}$ pump pulse of circular polarization.

There are two disadvantages if the pump pulse interacts with a nanoplasma gas instead of a uniform plasma. First, the ionization stage cannot be controlled as precisely as by optical-field ionization to produce a large population of lasing ion species. Second, the nanoplasma structure can disrupt EUV propagation and consequently hinder the amplification process. In our experimental study we measure the lasing intensity and the Rayleigh scattering from nanoplasmas as functions of the pump-pulse delay with respect to the prepulse. The results are shown in Fig. 2. With zero delay no lasing signal is observed as in the case without a prepulse. When the delay is increased the lasing signal increases and reaches a maximum at $3.8 \mathrm{~ns}$. After that the signal decreases gradually. The measurement of Rayleigh scattering intensity reveals that nanoplasmas created by the prepulse expand to the critical density on a time scale of ten picoseconds. With this measured expansion rate, the time scale for the mixing of individual nanoplasmas to form a uniform plasma is estimated from the average intercluster distance to be a few nanoseconds, and that for significant decrease of average density due to expansion of the whole plasma column to be around ten nanoseconds. It is also found that with higher prepulse intensity the nanoplasmas expand faster and the optimal pump-pulse delay decreases. These experimental observations suggest that the increase of the lasing signal with increasing delay is a result of increasing plasma uniformity and after the maximum the decrease with further delay is due to the reduction of on-axis ion density.

It is expected that a higher atom density should lead to a larger gain of amplification and thus larger lasing intensity. Figure 3 shows the dependence of EUV lasing intensity on average atom density. It is found that a maximum occurs at $3.3 \times 10^{17} \mathrm{~cm}^{-3}$. The simultaneous measurement of the plasma electron density distribution shows that the plasma column created by the pump pulse diverges as the atom density increased. This ionization-induced refraction results in lower pump intensity and thus shortens the gain length. The observation shows that the existence of an optimal atom density as previously reported [2,3] is the result of balance between a higher gain coefficient and a shorter gain length. 


\section{JThG5}

(a)

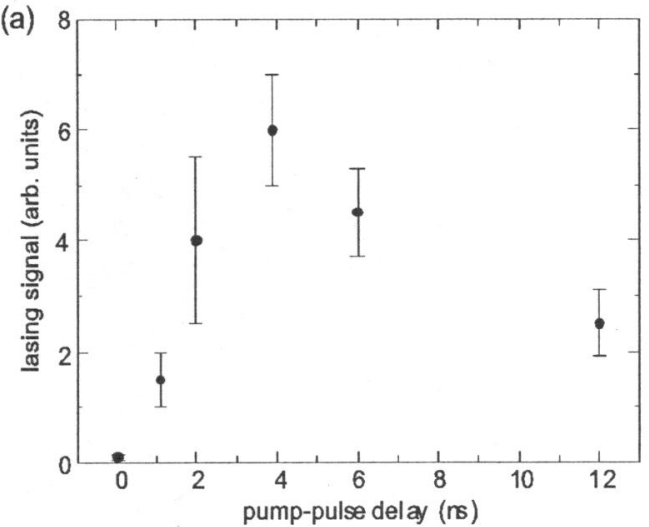

(b)

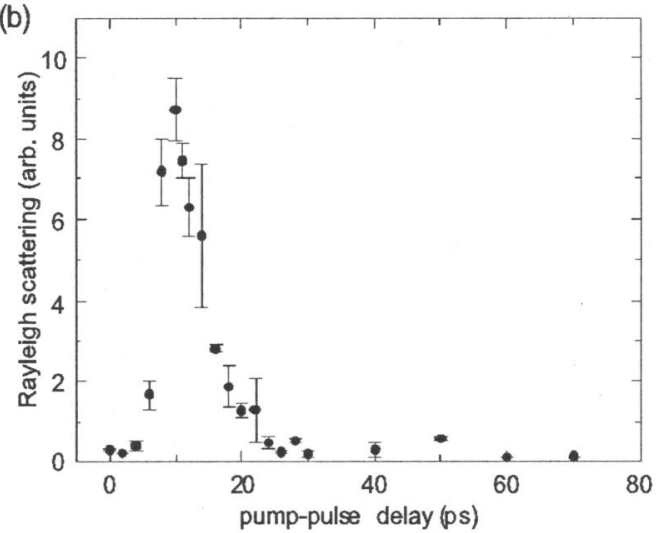

Fig. 2. Intensities of lasing signal (a) and Rayleigh scattering from nanoplasmas (b) as functions of pump-pulse delay. The peak intensities of the prepulse and the pump pulse are $1.5 \times 10^{14} \mathrm{~W} / \mathrm{cm}^{2}$ and $7 \times 10^{17} \mathrm{~W} / \mathrm{cm}^{2}$, respectively. The average atom density is $4 \times 10^{17} \mathrm{~cm}^{-3}$.

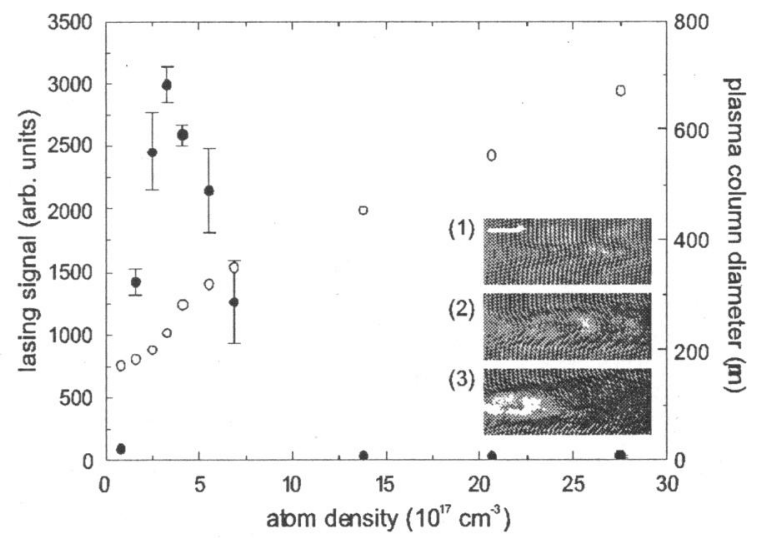

Fig. 3. Lasing intensity (solid circle) and the diameter of the plasma column produced by the pump pulse at $4 \mathrm{~mm}$ from the entrance (open circle) as functions of average atom density. Inset: interferograms of the plasma column taken at 50 ps after the pump pulse for (1) $3 \times 10^{17}$, (2) $1.3 \times 10^{18}$, and (3) $2.7 \times 10^{18}-\mathrm{cm}^{-3}$ atom densities.

\section{Reference}

[1] B. E. Lemoff et al., "Demonstration of a 10-Hz femtosecond-pulse-driven XUV laser at $41.8 \mathrm{~nm}$ in Xe IX," Phys. Rev. Lett. 74, 1574-1577 (1995).

[2] S. Sebban et al., "Saturated amplification of a collisionally pumped optical-field-ionization soft x-ray laser at $41.8 \mathrm{~nm}$," Phys. Rev. Lett. 86, 3004 (2001).

[3] S. Sebban et al., "Demonstration of a Ni-like Kr optical-field-ionization collisional soft x-ray laser at $32.8 \mathrm{~nm}$," Phys. Rev. Lett. 89, 253901 (2002).

[4] A. Butler et al., "Demonstration of a collisionally excited optical-field-ionization XUV laser driven in a plasma waveguide," Phys. Rev. Lett. 91, 205001 (2003)

[5] Ph. Zeitoun et al., "A high-intensity highly coherent soft X-ray femtosecond laser seeded by a high harmonic beam," Nature 431, 426 (2004).

[6] O. F. Hagena and W. Obert, "Cluster formation in expanding supersonic jets: effect of pressure, temperature, nozzle size, and test gas," J. Chem. Phys. 56, 1793 (1972).

[7] H.-H Chu et al., "A versatile 10-TW laser system with robust passive controls to achieve high stability and spatiotemporal quality," Appl. Phys. B 79, 193 (2004).

[8] P. B. Corkum, N. H. Burnett, and F. Brunel, “Above-threshold ionization in the long-wavelength limit," Phys. Rev. Lett. 62, 1259 (1989).

[9] S. Augst, D. D. Meyerhofer, D. Strickland, and S. L. Chin, "Laser ionization of noble gases by Coulomb-barrier suppression," J. Opt. Soc. Am. B 8, 858 (1991) 